\title{
Simulation of the Electric Field Distribution Near a Topographically Nanostructured Titanium-Electrolyte Interface: Influence of the Passivation Layer
}

\author{
Andreas Körtge, Patrick Elter, Regina Lange, and Ulrich Beck \\ Interface Research Group, Institute of Electronic Appliances and Circuits, University of Rostock, Albert-Einstein Straße 2, \\ 18059 Rostock, Germany
}

Correspondence should be addressed to Ulrich Beck; ulrich.beck@uni-rostock.de

Received 11 January 2013; Revised 15 April 2013; Accepted 15 April 2013

Academic Editor: Nageh K. Allam

Copyright (C) 2013 Andreas Körtge et al. This is an open access article distributed under the Creative Commons Attribution License, which permits unrestricted use, distribution, and reproduction in any medium, provided the original work is properly cited.

\begin{abstract}
A major challenge in biomaterials research is the regulation of protein adsorption at metallic implant surfaces. Recently, a number of studies have shown that protein adsorption can be influenced by metallic nanotopographies, which are discussed to increase electric field strengths near sharp edges and spikes. Since many metallic biomaterials form a native passivation layer with semiconducting properties, we have analyzed the influence of this layer on the near-surface electric field distribution of a nanostructure using finite element simulations. The Poisson-Boltzmann equation was solved for a titanium nanostructure covered by a $\mathrm{TiO}_{2}$ passivation layer in contact with a physiological $\mathrm{NaCl}$ solution (bulk concentration $0.137 \mathrm{~mol} / \mathrm{L}$ ). In contrast to a purely metallic nanostructure, the electric field strengths near sharp edges and spikes can be lower than in planar regions if a passivation layer is considered. Our results demonstrate that the passivation layer has a significant influence on the near-surface electric field distribution and must be considered for theoretical treatments of protein adsorption on passivated metals like titanium.
\end{abstract}

\section{Introduction}

Protein adsorption at the solid-liquid interface is one of the first steps of biomaterial-biosystem interaction after an implant is brought into contact with body fluid. In a number of studies it is concluded that the initially formed protein layer on a biomaterials surface is a key factor in influencing the subsequent cell attachment and the function of adherent cells [1-3]. Therefore, a detailed understanding of the adsorption process is of crucial importance. It is well established that various interaction types such as hydrophobic and entropic forces and electrostatic and dispersion interactions affect protein adsorption. However, it still remains to be elucidated to what extent each of these factors contributes to the overall process. In recent years, several studies have suggested that, besides various chemical functionalization methods, topographically nanostructured biomaterial surfaces are a promising approach for the regulation of protein adsorption. It was demonstrated that topographical nanostructures significantly affect the amount [3-6] and the biological function of adsorbed proteins [6-9]. Increased electric field strengths at sharp edges and spikes of the surface, geometric effects, and locally varying dispersion interactions are often discussed as possible explanations for this effect [10-12]. However, many medically relevant metallic implant materials form a passivation layer which separates the bulk metal from the wet environment of the biosystem. The physicochemical properties of these layers must therefore be considered, when discussing protein adsorption at most metallic implant surfaces. This is particularly important for titanium, one of the most commonly used materials for biomedical devices, whose excellent biocompatibility is attributed to its airformed passivation layer [13]. According to the literature, the amorphous film of stoichiometric $\mathrm{TiO}_{2}$ [14] shows semiconducting properties $[13,15,16]$. Consequently, compared to a metal-electrolyte interface, an additional space charge in the semiconductor must be considered in the discussion of the interface electrostatics [17]. The aim of this study was to investigate the influence of the passivation layer on the electric field 


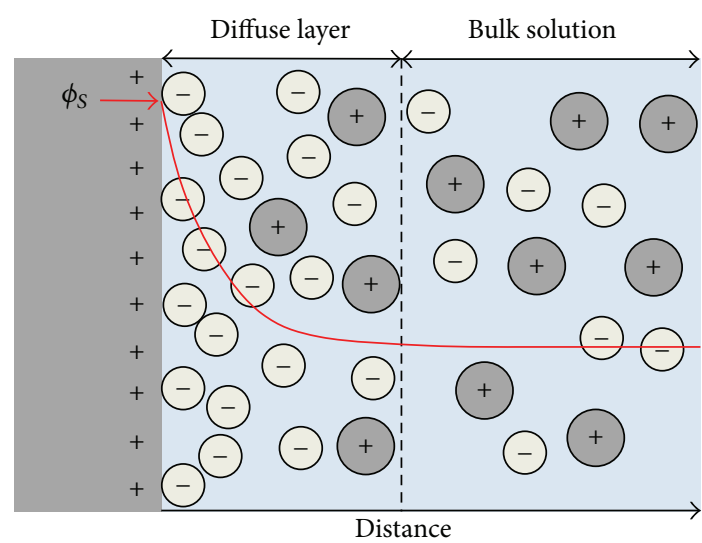

Figure 1: Counter ion distribution in a diluted symmetric $1: 1$ electrolyte solution near a charged surface.

distribution near a topographically nanostructured titaniumelectrolyte interface in an FEM simulation.

\section{Material and Methods}

2.1. The Electrolyte Solution Domain. Immediately after a metal is immersed into an electrolyte solution, an electrical double layer is formed; that is, the surface charge of the metal is compensated by an ionic counter charge [18]. For the description of the ion concentration distribution in diluted solutions, the Boltzmann distribution can be applied in the form

$$
c_{i}=c_{i}^{0} \exp \left(\frac{-q_{i} \phi}{k T}\right),
$$

with the ion concentration in the electrolyte bulk $c_{i}^{0}$, the ion charge $q_{i}$, the electric potential $\phi$, the Boltzmann constant $k$, and the absolute temperature $T$. Inserting expression (1) in Poisson's equation

$$
-\vec{\nabla}(\varepsilon \vec{\nabla} \phi)=\rho=\sum_{i} q_{i} c_{i}
$$

commonly with a constant permittivity $\varepsilon$, results in the classical nonlinear Poisson-Boltzmann equation, which adequately describes the electrical double layer for low electric surface potentials and low electrolyte concentrations. Figure 1 shows the course of the resulting electric potential and the distribution of ions in a diluted symmetric $1: 1$ electrolyte near a planar interface. The counter ions in the electrolyte screen the electric surface potential $\phi_{S}$, leading to an exponential decrease to zero in the solution bulk (Debye length $\approx 1 \mathrm{~nm}$ for a symmetric $0.1 \mathrm{M} 1: 1$ electrolyte [18]). However, the treatment of ions as point-like charges can lead to unrealistically high ion densities near the surface, when exceeding a certain electric surface potential [19]. Therefore, we use a modified ion concentration distribution proposed by Kilic et al. [19] which considers steric effects:

$$
c_{i}=\frac{c_{i}^{0} \exp \left(-q_{i} \phi / k T\right)}{1+2 \nu \sinh \left(\left|q_{i}\right| \phi / 2 k T\right)^{2}},
$$

where $v=2 a^{3} c_{i}^{0}$ is the packing parameter with $a$ as the effective ion size. We consider $a$ as the Stokes diameter of the hydrated electrolyte ions $d_{i}$, which leads to the ionic space charge density of a symmetric electrolyte solution:

$$
\rho_{\mathrm{El}}=\sum_{i=1}^{2} \frac{q_{i} c_{i}^{0} \exp \left(-q_{i} \phi / k T\right)}{1+4 \cdot d_{i}^{3} c_{i}^{0} \sinh \left(\left|q_{i}\right| \phi / 2 k T\right)^{2}} .
$$

2.2. Influence of the $\mathrm{TiO}_{2}$ Passivation Layer on the Interface Electrostatics. In a number of studies, it has been concluded that the passivation layer of titanium behaves like an n-type semiconductor with oxygen vacancies as main donor species $[13,15,16,20]$. In contact with an electrolyte solution, a potential difference is formed over the interface, just as in the case of a metal-electrolyte system. However, the conductivity of semiconductors is usually below that of an electrolyte, so that the potential drop takes place predominantly in the solid and leads to band bending. Therefore, when dealing with the semiconductor-electrolyte interface, an additional diffuse space charge layer in the solid must be considered [17].

For reasons of simplification we introduce a number of assumptions for our semiconductor model: (i) the $\mathrm{TiO}_{2}$ has an ideal electronic band structure, (ii) the system is in kinetic equilibrium; that is, the Fermi energies are aligned, (iii) surface states at the solid-liquid boundary of the $\mathrm{TiO}_{2}$ layer are neglected, (iv) the semiconductor is regarded as nondegenerate, $(\mathrm{v})$ the transition from the titanium to the $\mathrm{TiO}_{2}$ layer is abrupt, and (vi) the formation of an inversion layer, that is, the accumulation of minority charge carriers, is excluded because of the considered low band bending.

The investigated physicochemical situation is illustrated by means of a planar titanium $\mathrm{NaCl}$ solution interface. The liquid is separated from the metal by the $\mathrm{TiO}_{2}$ passivation layer with a thickness of approximately 3 to $10 \mathrm{~nm}$ [21] (Figure 2). In kinetic equilibrium the potential drop over the interface is expressed by

$$
\Delta \phi_{\text {Ti-Body }}=\frac{E_{\mathrm{Ti}}-E_{\text {Body }}}{e_{0}},
$$

where $E_{\mathrm{Ti}}$ is the work function of titanium and $E_{\mathrm{Body}}$ is the reference electron energy level in the bulk electrolyte, which is equal to the normal redox potential of the body fluid under physiological conditions $[16,22]$. The course of the electric potential in the passivation layer corresponds to the band bending and is related to the energy of the bottom conduction band edge in the semiconductor bulk $E_{\mathrm{C}_{\mathrm{TiO}}}^{B}$ (Figure 2).

When only considering the spatially varying electron density $n$, the space charge density in the semiconductor $\rho_{\mathrm{TiO}_{2}}$ is connected to the electric potential by the expression [18]

$$
\begin{aligned}
\rho_{\mathrm{TiO}_{2}} & =e_{0} \cdot\left(N_{D}-n\right) \\
& =e_{0} N_{D}\left[1-\exp \left(\frac{\phi-\phi_{\mathrm{TiO}_{2}}}{k T}\right)\right],
\end{aligned}
$$

with the maximum density of oxygen vacancies $N_{D}$, and the electric potential in the flat band case $\phi_{\mathrm{TiO}_{2}} \cdot \phi_{\mathrm{TiO}_{2}}$, relative to 


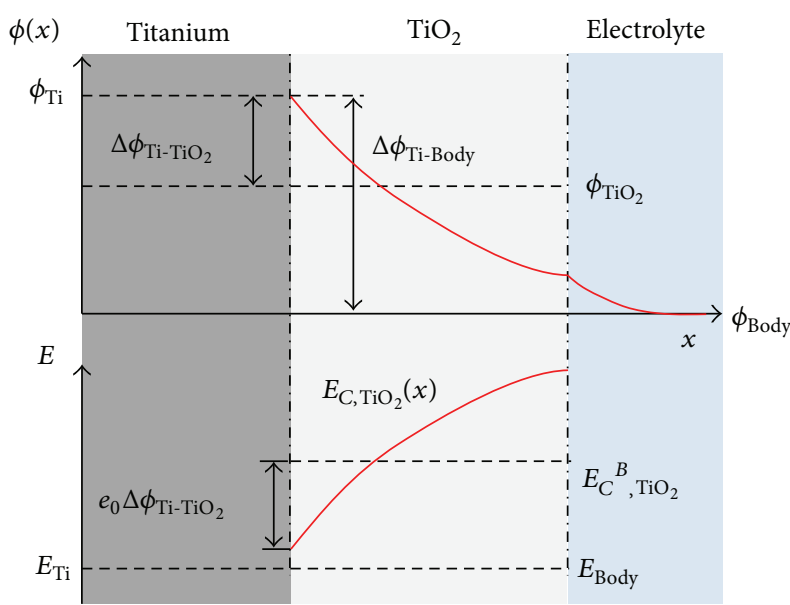

FIgURE 2: Course of the electric potential and the bottom conduction band of the $\mathrm{TiO}_{2}$ passivation layer over a planar titaniumelectrolyte interface in kinetic equilibrium. The Galvani potential drop $\Delta \phi_{\text {Ti-Body }}$ is induced by the difference of the titanium work function $E_{\mathrm{Ti}}$ and the reference electron energy level in the bulk solution $E_{\text {Body }}$.

the electric potential at the titanium surface $\phi_{\mathrm{Ti}}$, is given by the built-in potential difference $\Delta \phi_{\mathrm{Ti}-\mathrm{TiO}_{2}}$ :

$$
\begin{aligned}
\phi_{\mathrm{TiO}_{2}} & =\phi_{\mathrm{Ti}}+\Delta \phi_{\mathrm{Ti}^{-\mathrm{TiO}_{2}}} \\
& =\phi_{\mathrm{Ti}}+\frac{E_{\mathrm{Ti}}-E_{\mathrm{TiO}_{2}}}{e_{0}} \\
& =\phi_{\mathrm{Ti}}+\frac{E_{\mathrm{Ti}}-\chi_{\mathrm{TiO}_{2}}-\left(E_{C, \mathrm{TiO}_{2}}^{B}-E_{F, \mathrm{TiO}_{2}}\right)}{e_{0}} \\
& =\phi_{\mathrm{Ti}}+\frac{E_{\mathrm{Ti}}-\chi_{\mathrm{TiO}_{2}}-k T \ln \left(N_{C} / N_{D}\right)}{e_{0}},
\end{aligned}
$$

where $E_{\mathrm{TiO}_{2}}$ is the theoretical work function of $\mathrm{TiO}_{2} \cdot E_{\mathrm{TiO}_{2}}$ is the sum of the $\mathrm{TiO}_{2}$ electron affinity $\chi_{\mathrm{TiO}_{2}}$ and the energy gap between the lower conduction band edge and the Fermi energy $\left(E_{C, \mathrm{TiO}_{2}}^{B}-E_{F, \mathrm{TiO}_{2}}\right)$ which is equal to $k T \ln \left(N_{C} / N_{D}\right)$ with the effective density of states of electrons in the conduction band $N_{C}=2\left(2 \pi m_{e}{ }^{*} k T / h^{2}\right)^{3 / 2}$, Planck's constant $h$, and the effective mass of electrons in the $\mathrm{TiO}_{2}$ layer $m_{e}^{*}$. Finally, inserting (7) in (6) yields the space charge density in the $\mathrm{TiO}_{2}$ passivation layer:

$$
\begin{aligned}
\rho_{\mathrm{TiO}_{2}}= & e_{0} N_{D} \\
& \times\left[1-\exp \left(\frac{e_{0}\left(\phi-\phi_{\mathrm{Ti}}\right)+\chi_{\mathrm{TiO}_{2}}+k T \ln \left(N_{C} / N_{D}\right)-E_{\mathrm{Ti}}}{k T}\right)\right] .
\end{aligned}
$$

Another important point in the discussion of the titanium-electrolyte interface is the $\mathrm{pH}$-dependent surface charge of $\mathrm{TiO}_{2}$. Titanium hydroxides are amphoteric; that is, they show both acidic and basic properties because of their two different types of coordination to the surface titanium cations [13]. In contact with an aqueous electrolyte solution,

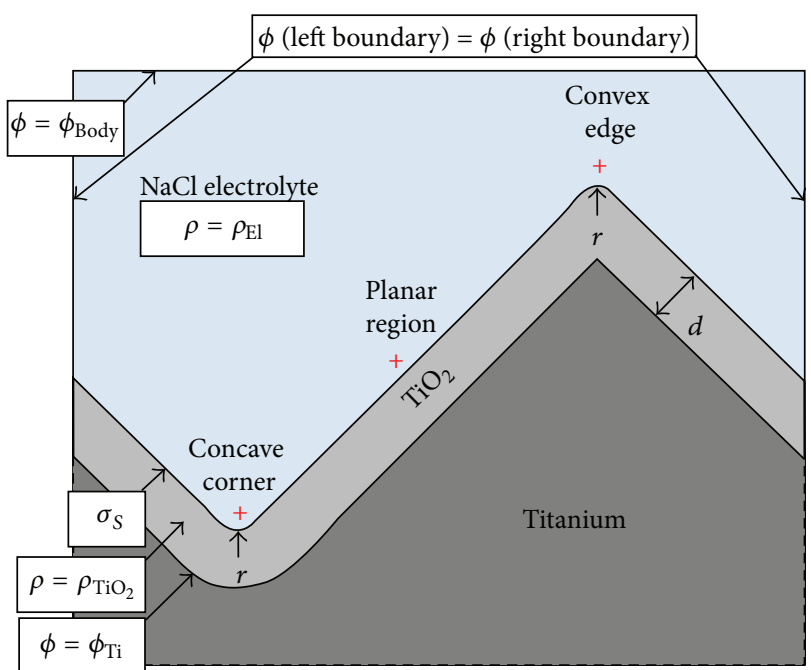

FIGURE 3: Cross-section of the grooved titanium-electrolyte interface with the used boundary conditions. The titanium is covered by a $\mathrm{TiO}_{2}$ passivation layer with thickness $d$. The designations concave corner, planar region, and convex edge refer to the positions of the red crosses. $r$ defines the radius of curvature of the $\mathrm{TiO}_{2}$-electrolyte boundary.

this amphoteric behavior results in the $\mathrm{pH}$-dependent formation of negatively and positively charged hydroxides that are considered in the literature as potential determining ions [23]. We assume that the point of zero charge of $\mathrm{TiO}_{2}$, that is, the $\mathrm{pH}$ value at which the positive and negative charges at the surface cancel each other, is close to neutrality [13]. According to Ridley et al. [24], the physiologically relevant $\mathrm{pH}$ range from 5 to 7.5 [25] is therefore equivalent to a surface charge density $\sigma_{S}$ ranging from 0.05 to $-0.05 \mathrm{C} / \mathrm{m}^{2}$.

2.3. Finite Element Model. FEM-based electrostatics simulations were conducted with COMSOL Multiphysics (v4.2, COMSOL AG) using triangular elements and the multifrontal massively parallel sparse direct solver (MUMPS). The mesh was refined until the relative error (a posteriori) of the electric field strength in the electrolyte near the planar area of the surface was below $1 \%$ resulting in approximately $3.2 \cdot 10^{5}$ triangular elements after four mesh refinements (see Supplementary Material available online at http://dx.doi.org/10.1155/2013/820914). The iteration was stopped when the relative error estimate for each nonlinear iteration step was below $1 \cdot 10^{-6}$.

Figure 3 shows the 2D simulation box of the topographically nanostructured titanium $\mathrm{NaCl}$ electrolyte interface. A grooved nanostructure was chosen as model geometry, because it represents the simplest topographical variation of a surface which, however, contains the majority of geometric features: concave corners, convex edges, and planar structure elements. Furthermore, the complexity of the problem and the computational effort can be reduced by treating a $2 \mathrm{D}$ cross-section of a groove. The model geometry consists of three planar regions, a $90^{\circ}$ concave corner, and a $90^{\circ}$ convex edge with the radius of curvature of the solid-liquid phase boundary $r$. We considered a passivation layer with a constant 


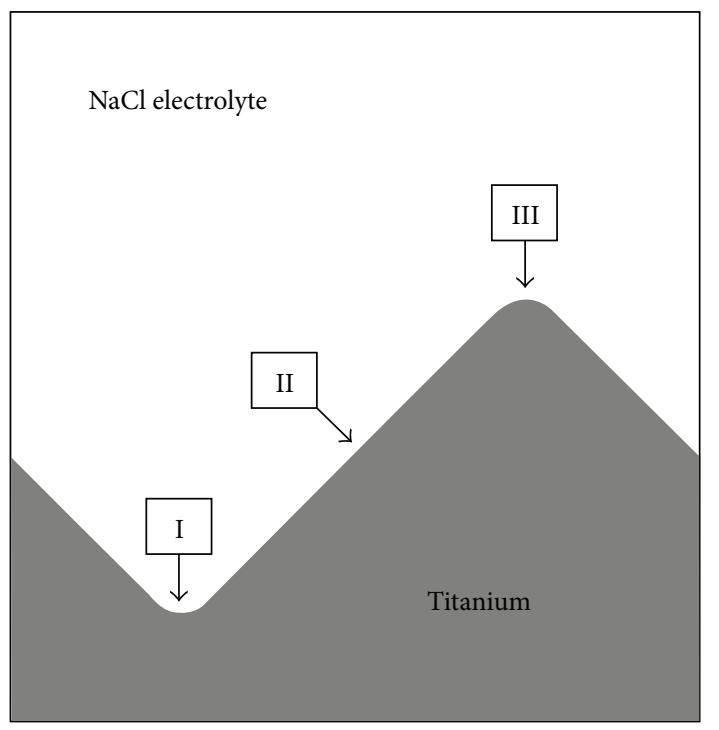

(a)

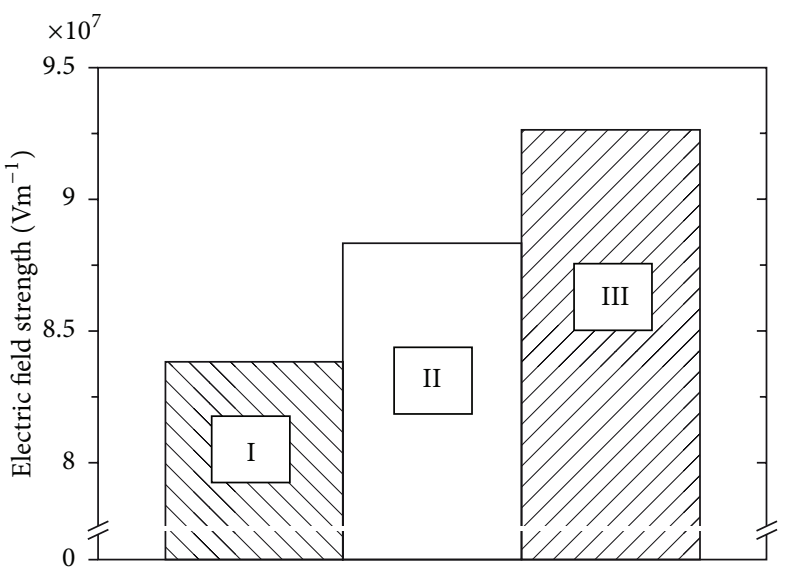

(b)

FIGURE 4: Cross-section of the grooved metal surface in contact with an $\mathrm{NaCl}$ electrolyte solution. The values of the electric field strength in $0.3 \mathrm{~nm}$ distance from the solid-liquid boundary at the concave corner (I), the planar region (II), and the convex edge (III) are given in (b).

thickness $d$, which is in contact with a physiological $\mathrm{NaCl}$ electrolyte solution with a bulk concentration of $0.137 \mathrm{~mol} / \mathrm{L}$. The growth of the $\mathrm{TiO}_{2}$ layer is assumed to occur only in direction of the titanium bulk because of the unique oxide mechanism of this metal $[15,26,27]$. The growth of a film with thickness $d$ therefore results in an edge radius of $r+d$ at the concave corner of the $\mathrm{TiO}_{2}$-titanium boundary. Depending on the ratio of $r$ and $d$, two cases must be differentiated for the convex edge of the $\mathrm{TiO}_{2}$-titanium boundary: (i) $r / d>1$ : the radius of the convexly curved boundary is $r-d$ and (ii) $r / d \leq 1$ : a sharp convex edge is formed. In the latter case, a radius of $0.2 \mathrm{~nm}$ (approximate atom radius of titanium) was subsequently inserted to avoid numerical problems.

$\phi_{\mathrm{Ti}}$ was applied at the titanium- $-\mathrm{TiO}_{2}$ interface using Dirichlet boundary conditions. In the bulk solution the electric potential decreases to the body potential $\phi_{\text {Body }}$ (top boundary: $\left.\phi=\phi_{\text {Body }}\right)$. The geometry allows applying periodic boundaries conditions at the left and the right boundary. Neumann boundary conditions were used for the $\mathrm{pH}$ dependent surface charge $\sigma_{S}$ at the $\mathrm{TiO}_{2}$-electrolyte phase boundary. The space charge densities in the electrolyte and the $\mathrm{TiO}_{2}$ domain are described by (4) and (8), respectively.

To facilitate a comparison to the metal-semiconductorelectrolyte system, we also introduce a simple metalelectrolyte model. In this case the position of the solid-liquid interface was fixed and the passivation layer thickness $d$ was set to zero. In this reference model a constant electric potential $\phi_{S}$ was applied at the metal-electrolyte phase boundary using Dirichlet boundary conditions.

2.4. Model Parameters. The parameters used in this study are listed in Table 1. For some parameters multiple values are given in the cited literature $\left(\varepsilon_{\mathrm{TiO}_{2}}, \phi_{\mathrm{Body}}, N_{D}, d, \sigma_{S}\right)$. The surface charge density $\sigma_{S}$ was varied according to the physiologically relevant $\mathrm{pH}$ range. Mean values of the other parameters
TABLE 1: Overview of the used parameter values.

\begin{tabular}{lcc}
\hline Parameter & Value & Source \\
\hline$c_{i}^{0}(\mathrm{~mol} / \mathrm{L})$ & 0.137 & \\
$T(\mathrm{~K})$ & 25 & {$[28]$} \\
$\varepsilon_{\mathrm{El}}$ & 79 & {$[23]$} \\
$\varepsilon_{\mathrm{TiO}_{2}}$ & $48,78,110$ & {$[29]$} \\
$d_{\mathrm{Na}^{+}}(\mathrm{nm})$ & 0.368 & {$[29]$} \\
$d_{\mathrm{Cl}^{-}}(\mathrm{nm})$ & 0.242 & {$[30]$} \\
$E_{\mathrm{Ti}}(\mathrm{eV})$ & 4.1 & {$[31]$} \\
$E_{\mathrm{SCE}}(\mathrm{eV})$ & 4.75 & {$[32]$} \\
$\chi_{\mathrm{TiO}}(\mathrm{eV})$ & 4.04 & {$[33]$} \\
$m_{e}^{*}\left(\mathrm{~m}_{\mathrm{e}}\right)$ & 5.6 & {$[34]$} \\
$\phi_{\mathrm{S}}(\mathrm{mV})$ & -100 & {$[24]$} \\
$\sigma_{S}\left(\mathrm{C} / \mathrm{m}^{2}\right)$ & $-0.05,0,0.05$ & {$[22]$} \\
$\phi_{\mathrm{Body}}\left(\mathrm{mV} \mathrm{SCE}_{\mathrm{SE}}\right)$ & $350,450,550$ & {$[16]$} \\
$N_{D}\left(10^{20} \mathrm{~cm}^{-3}\right)$ & $1,1.5,2$ & {$[21]$} \\
$d(\mathrm{~nm})$ & $3,5,10$ & \\
$r(\mathrm{~nm})$ & $1,3,5$ &
\end{tabular}

were used for the results shown below. Furthermore, sensitivity studies were conducted for $\varepsilon_{\mathrm{TiO}_{2}}, \phi_{\mathrm{Body}}, N_{D}, d$, and $r$ in order to estimate the influence of the respective parameter on the results (see supplementary material). The electric potential at the titanium- $\mathrm{TiO}_{2}$ boundary $\phi_{\mathrm{Ti}}$ and the potential in the $\mathrm{TiO}_{2}$ layer in the flat band case $\phi_{\mathrm{TiO}_{2}}$ are calculated using (5) and (7), respectively, and amount to $\phi_{\mathrm{Ti}}=1.10 \mathrm{~V}$ and $\phi_{\mathrm{TiO}_{2}}=1.14 \mathrm{~V}$.

\section{Results and Discussion}

3.1. The Metal-Electrolyte Interface. Figure 4(a) shows a schematic representation of the topographically nanostructured 

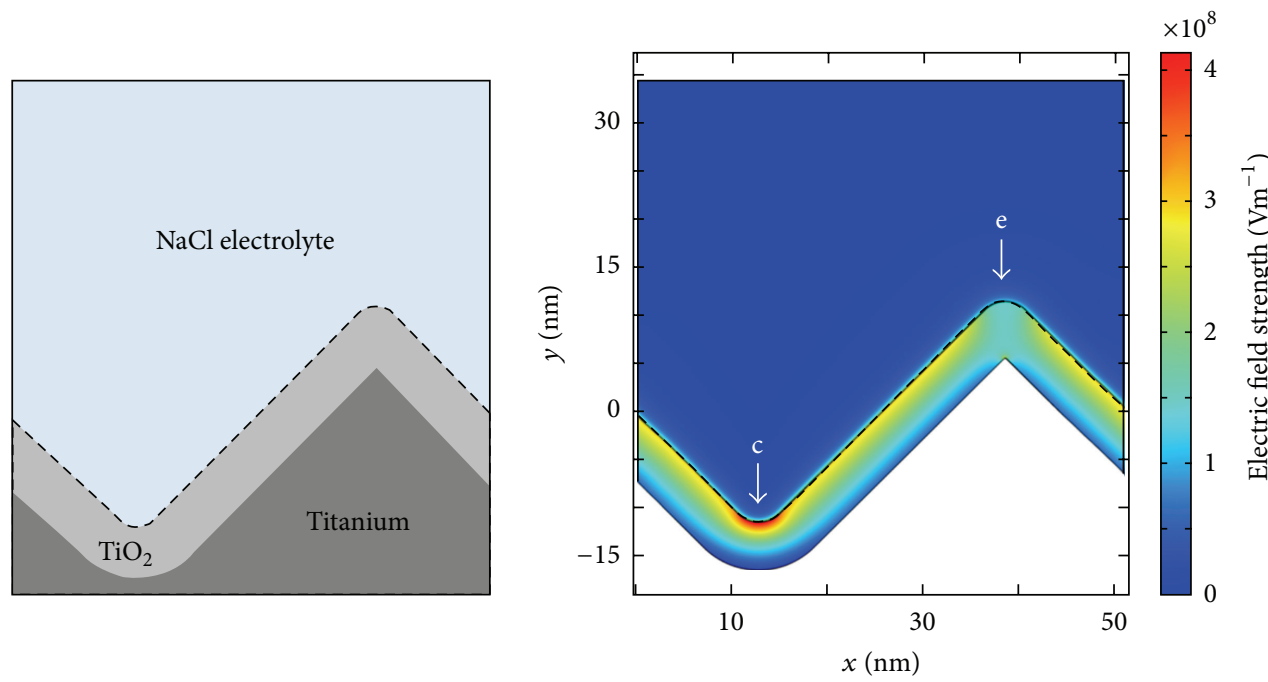

(a)

(b)

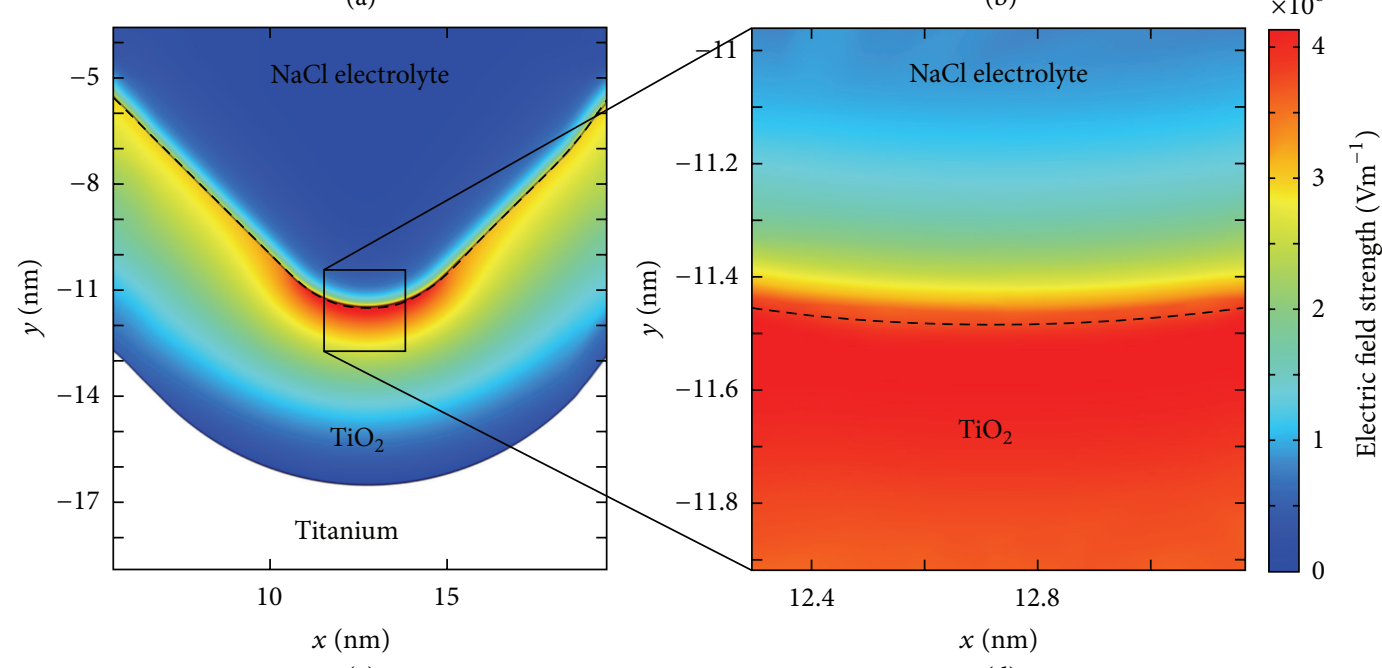

(c)

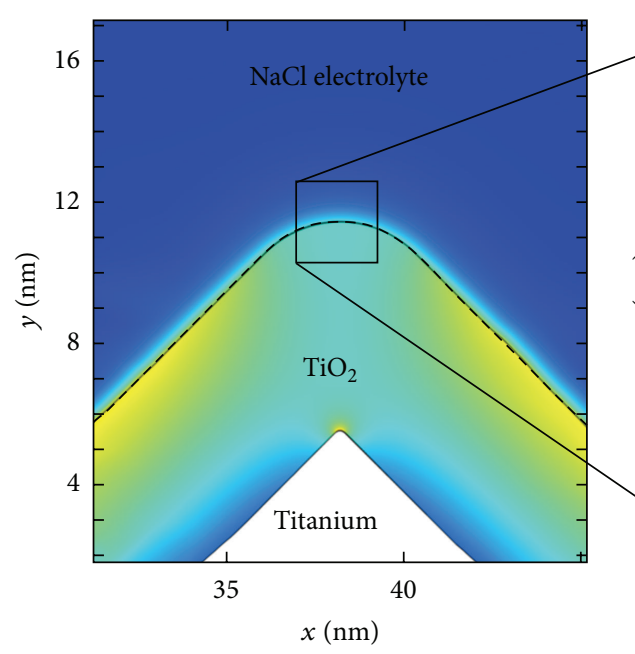

(e) (d)

$\times 10^{8}$

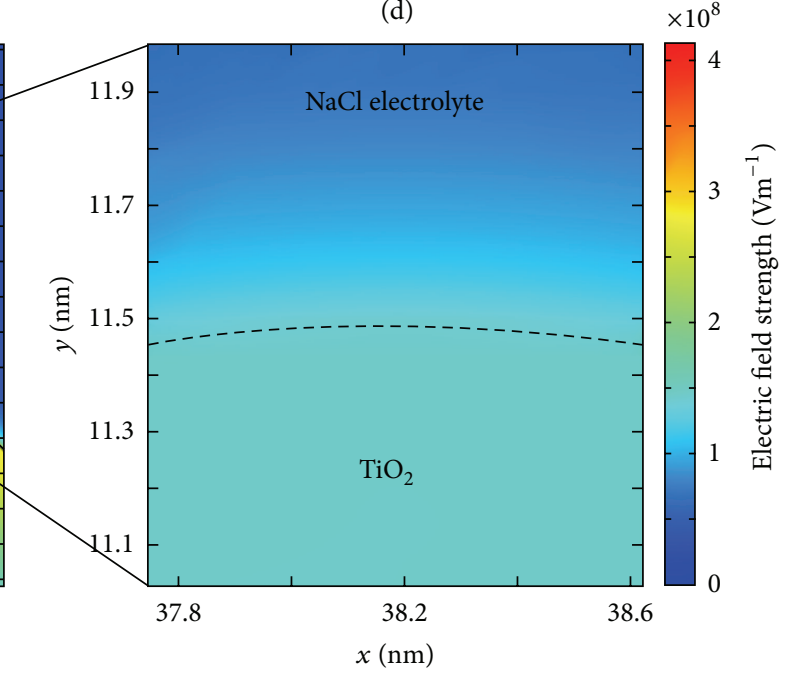

(f)

FIGURE 5: (a) Cross-section of the passivated titanium nanostructure in contact with an $\mathrm{NaCl}$ electrolyte solution. (b) Field strength distribution of the titanium-electrolyte interface. (c) Magnification of the concave corner. (d) Detail of the concave corner. (e) Magnification of the convex edge. (f) Detail of the convex edge. 
metal-electrolyte interface. The position-dependent electric field variation in the $\mathrm{NaCl}$ electrolyte is compensated within a few nanometers distance from the surface (Debye length $<1 \mathrm{~nm}$ ) because of strong ionic shielding. Therefore, the field strength was determined at the concave corner, the planar region, and the convex edge in $0.3 \mathrm{~nm}$ distance from the solid-liquid phase boundary (Figure 4(b)). Compared to the position near the planar surface (II), the electric field strength is increased at the concave corner (I) and decreased at the convex edge (III) by approximately $5 \%$. This relatively small variation can also be attributed to the ionic space charge in the electrolyte. A comparative simulation with a $0.01 \mathrm{M} \mathrm{NaCl}$ electrolyte solution showed a $15 \%$ increase and a $20 \%$ decrease compared to the planar region at the convexly and the concavely curved boundaries, respectively. The consideration of a grooved nanostructure is another reason for the slight electric field variation, because the surface curvature only occurs in two dimensions. We expect a more strongly pronounced influence of topography on the local electric field distribution at 3D structures, for instance, pits and spikes.

3.2. The Titanium-TiO ${ }_{2}$-Electrolyte Interface. Figure 5(a) shows the simulation box of the topographically nanostructured titanium-electrolyte interface. The system consists of the bulk titanium which is covered by a $5 \mathrm{~nm}$ thick $\mathrm{TiO}_{2}$ passivation layer and the adjacent $\mathrm{NaCl}$ electrolyte solution. The corresponding distribution of the electric field strength allowing for the semiconducting passivation layer is depicted in Figure 5(b). The range of the electric field in the semiconductor is considerably larger than in the electrolyte because of the comparatively low charge density. Similar to the metal-electrolyte interface, the strong decrease of the electric field strength in the electrolyte occurs within a few nanometers distance from the solid-liquid phase because of the ionic shielding. Therefore, the concavely and the convexly curved area of the interface are depicted greatly magnified in Figures 5(c)-5(d) and Figures 5(e)-5(f), respectively. It is apparent from a comparison of Figures 5(d) and 5(f) that the electric field distribution in close proximity to the $\mathrm{TiO}_{2}$ electrolyte phase boundary is inverted in comparison to the pure metal-electrolyte system (Figure 4(b)): the electric field at the concave corner is significantly stronger than at the convex edge.

Values of the electric field strength near the curved and the planar boundary elements in $0.3 \mathrm{~nm}$ distance from the solid-liquid phase boundary are given in Figure 6 for three $\mathrm{pH}$ values. At $\mathrm{pH} 5$ the electric field strength at the concavely and the convexly curved boundaries is approximately increased by $10 \%$ and decreased by $20 \%$, respectively, compared to the planar region. The inversion is maintained over the entire considered $\mathrm{pH}$ range and it is most strongly pronounced near the physiological $\mathrm{pH}$ value, where an increase of $20 \%$ and a decrease of $60 \%$ are observed at the concave corner and the convex edge, respectively.

In order to understand the behavior of the electric field described above, the surface equipotential lines at the convex edges of a metal without a passivation layer and of passivated titanium are schematically depicted in Figure 7.
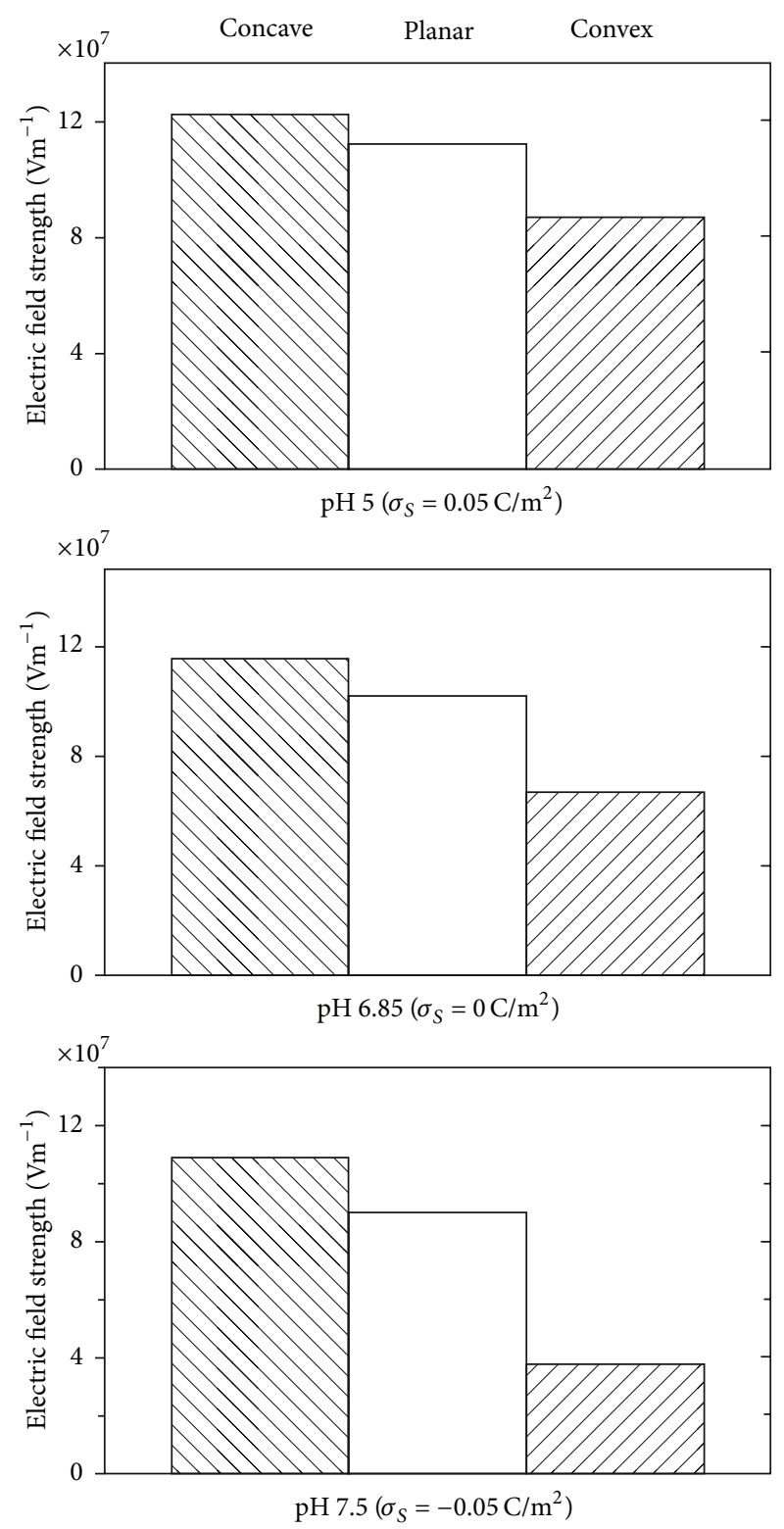

FIGURE 6: Values of the electric field strength in the $\mathrm{NaCl}$ electrolyte in $0.3 \mathrm{~nm}$ distance from the $\mathrm{TiO}_{2}$-electrolyte boundary for three $\mathrm{pH}$ values.

The equipotential line at the metal surface strictly follows the geometry (Figures $7(\mathrm{a})-7(\mathrm{~b})$ ); that is, the surface potential is constant. This potential distribution results in increased electric field strengths at convex edges because of greater field line densities. Regarding the semiconducting passivation layer of titanium, this typically metallic characteristic changes: the equipotential line of the $\mathrm{TiO}_{2}$ surface cannot follow the geometry. The surface potential of the $\mathrm{TiO}_{2}$ film therefore depends on the curvature of the interface (Figures 7 (c)-7(d)) and in case of the convex edge this leads to a locally decreased electric potential.

Figure 8 illustrates how this altered electric potential distribution affects the electric field strengths near the topographically nanostructured titanium-electrolyte interface by means of cut lines at the curved and planar regions of 


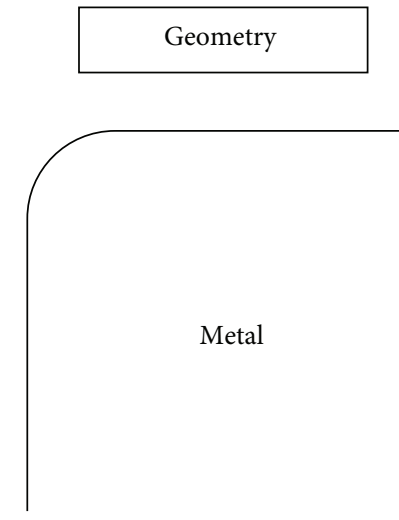

(a)

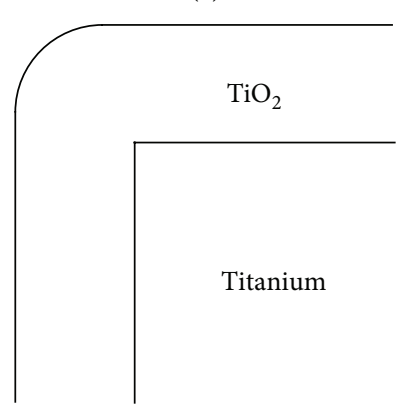

(c)
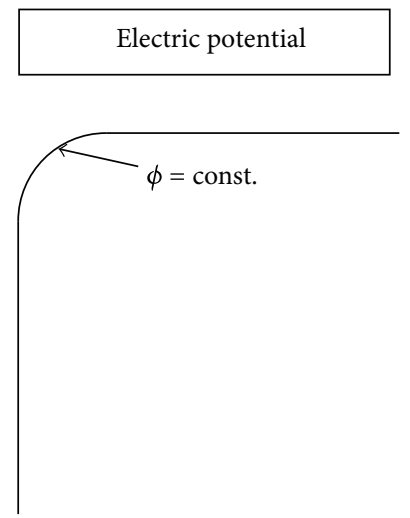

(b)

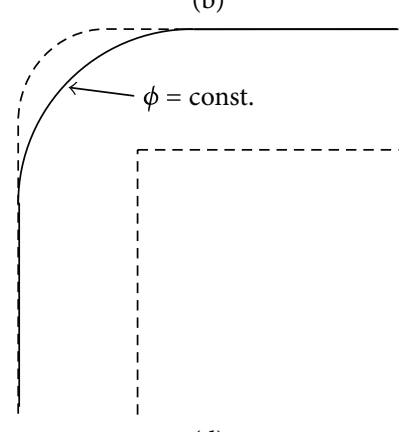

(d)
FIGURE 7: Schematic illustration of (a) the geometry of the convex edge of a metal nanotopography, (b) the equipotential line of the metal surface, (c) the geometry of the convex edge of the $\mathrm{TiO}_{2}$ covered titanium nanotopography, and (d) an equipotential line in the semiconductor layer.

the interface (Figure 8(a)). The courses of the potential and the corresponding field strength along these cut lines are depicted in Figures 8(b) and 8(c), respectively. In the concave corner (black cut line) the potential in the $\mathrm{TiO}_{2}$ decreases less strongly compared to the planar region (red cut line), whereas it decreases more strongly near the convex edge (green cut line). The electric field strengths along the cut lines according to the courses of the electric potential are depicted in Figure 8(c). As mentioned above, considerable differences of the local electric field strength in the electrolyte only occur very close to the interface. The locally varying electric potential induces varyingly strong ionic shielding, and differences are compensated in approximately $2 \mathrm{~nm}$ distance from the solid surface.

Recently, a number of studies concerning the adsorption behavior of biomolecules on both stochastically and regularly topographically nanostructured biomaterial surfaces have reported a significantly increased amount of adsorbed proteins in comparison to planar surfaces [3-6]. Moreover, spatially resolved measurements by means of scanning forcemicroscopic methods facilitated the identification of preferential adsorption sites at concave regions of the nanotopographies $[3,6]$. Our simulations predict the highest electric field strengths near concave regions of a passivated titanium

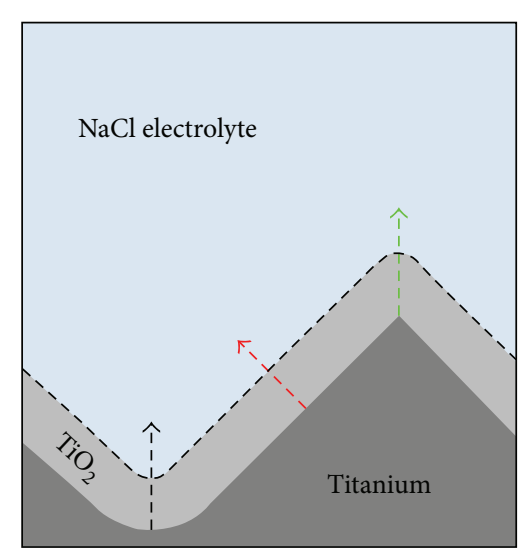

(a)
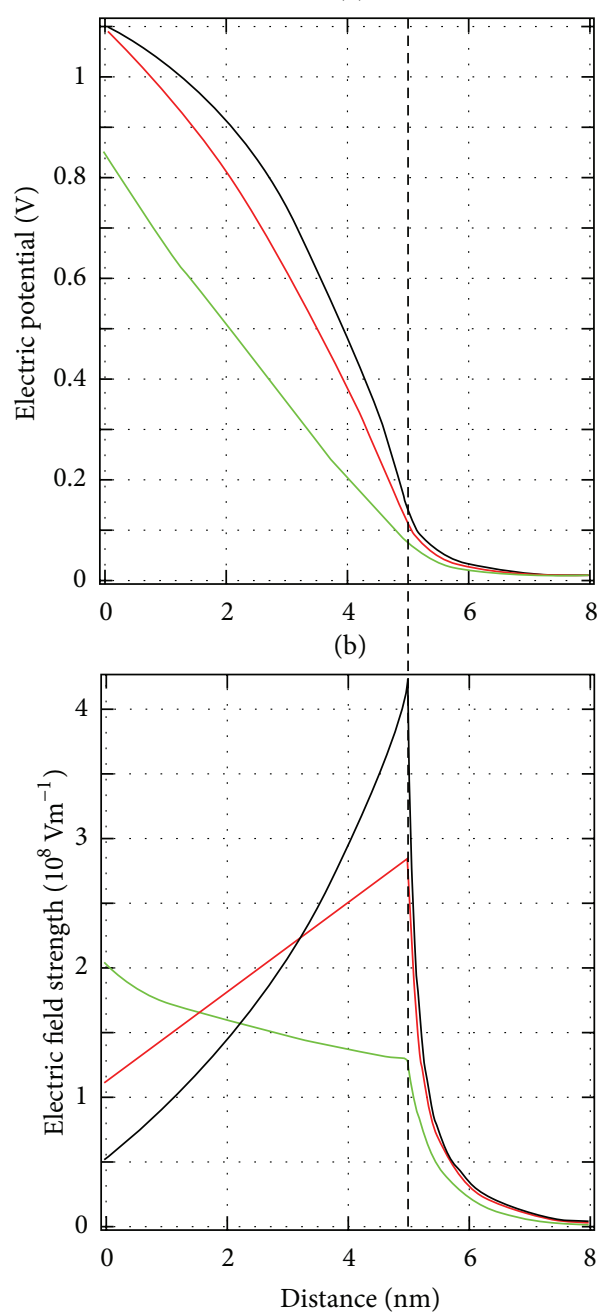

(c)

Figure 8: (a) Cross-section of the grooved titanium-electrolyte interface with cut lines perpendicular to the surface at the convex corner (black), the planar region (red), and the convex edge (green). (b) Course of the electric potential along cut lines perpendicular to the interface. (c) Course of the electric field strength perpendicular to the interface. 
surface. This result suggests that the increased amount of adsorbed biomolecules in these regions, among others, may be caused by the increased electric field strengths.

\section{Conclusion}

In this study, the influence of the air-formed $\mathrm{TiO}_{2}$ passivation layer on the electric field distribution near a topographically nanostructured titanium-electrolyte interface was investigated in a finite element-based simulation. Compared to a planar region of the interface, increased and decreased electric field strengths were determined near the concave and convex regions, respectively, allowing for the semiconducting film. This constitutes an inversion of the electric field strength distribution near the reference metal-electrolyte interface, where the highest electric field strengths occur near the convexly curved region. Although the absolute values of the field strength are altered by the $\mathrm{pH}$-dependent surface charge, particularly at the convex edge, the inversion is maintained over the entire considered $\mathrm{pH}$ range. Therefore, the band bending in the semiconducting $\mathrm{TiO}_{2}$ layer is regarded as the main factor determining the characteristic near-surface electric field strength distribution of the passivated titaniumelectrolyte interface. Our results may provide a possible explanation for the increased accumulation of proteins at concave regions of the interface as it was observed in a number of experimental and computational studies on the influence of the electrostatic interaction on protein adsorption at topographical nanostructures.

\section{Acknowledgment}

Funding from the Deutsche Forschungsgemeinschaft DFG (Graduate School "Welisa" 1505/2 and Project BE 2362/2-2) is gratefully acknowledged.

\section{References}

[1] K. Anselme, "Osteoblast adhesion on biomaterials," Biomaterials, vol. 21, no. 7, pp. 667-681, 2000.

[2] C. J. Bettinger, R. Langer, and J. T. Borenstein, "Engineering substrate topography at the micro- and nanoscale to control cell function," Angewandte Chemie International Edition, vol. 48, pp. 5406-5415, 2009.

[3] C. González-García, S. R. Sousa, D. Moratal, P. Rico, and M. Salmerón-Sánchez, "Effect of nanoscale topography on fibronectin adsorption, focal adhesion size and matrix organisation," Colloids and Surfaces B, vol. 77, pp. 181-190, 2010.

[4] C. Galli, M. Collaud Coen, R. Hauert et al., "Protein adsorption on topographically nanostructured titanium," Surface Science, vol. 474, no. 1-3, pp. L180-L184, 2001.

[5] I. Salakhutdinov, P. Vandevord, O. Palyvoda et al., "Fibronectin adsorption to nanopatterned silicon surfaces," Journal of Nanomaterials, vol. 2008, Article ID 543170, 5 pages, 2008.

[6] P. Elter, R. Lange, and U. Beck, "Atomic force microscopy studies of the influence of convex and concave nanostructures on the adsorption of fibronectin," Colloids and Surfaces, vol. 89, pp. 139-146, 2012.
[7] F. A. Denis, P. Hanarp, D. S. Sutherland et al., "Protein adsorption on model surfaces with controlled nanotopography and chemistry," Langmuir, vol. 18, no. 3, pp. 819-828, 2002.

[8] P. Roach, D. Farrar, and C. C. Perry, "Interpretation of protein adsorption: surface-induced conformational changes," Journal of the American Chemical Society, vol. 127, pp. 8168-8173, 2005.

[9] P. Roach, D. Farrar, and C. C. Perry, "Surface tailoring for controlled protein adsorption: effect of topography at the nanometer scale and chemistry," Journal of the American Chemical Society, vol. 128, no. 12, pp. 3939-3945, 2006.

[10] P. Elter and R. Thull, "Computer Simulations: modeling of protein adsorption on nanostructured metallic surfaces," in Metallic Biomaterial Interfaces, J. Breme, R. Thull, and C. J. Kirkpatrick, Eds., pp. 163-169, Wiley-VCH, Weinheim, Germany, 2008.

[11] P. Elter, R. Lange, and U. Beck, "Electrostatic and dispersion interactions during protein adsorption on topographic nanostructures," Langmuir, vol. 27, no. 14, pp. 8767-8775, 2011.

[12] E. Gongadze, D. D. Kabaso, S. Bauer et al., "Adhesion of osteoblasts to a nanorough titanium implant surface," International Journal of Nanomedicine, vol. 2011, pp. 1801-11816, 2011.

[13] M. Textor, C. Sittig, V. M. Frauchiger, S. Tosatti, and D. M. Brunette, "Properties and biological significance of natural oxide films on titanium and its alloys," in Titanium in Medicine: Material Science, Surface Science, Engineering, Biological Responses and Medical Applications, D. M. Brunette, P. Tengvall, M. Textor, and P. Thomsen, Eds., pp. 171-230, Springer, Berlin, Germany, 2001.

[14] J. E. G. González and J. C. Mirza-Rosca, "Study of the corrosion behavior of titanium and some of its alloys for biomedical and dental implant applications," Journal of Electroanalytical Chemistry, vol. 471, pp. 109-115, 1999.

[15] T. Hurlen and S. Hornkjøl, "Anodic growth of passive films on titanium," Electrochimica Acta, vol. 36, no. 1, pp. 189-195, 1991.

[16] D. Scharnweber, R. Beutner, S. Rößler, and H. Worch, "Electrochemical behaviour of titanium based materials-are there relations to biocompatibility?" Journal of Materials Science, vol. 13, pp. 1215-1220, 2002.

[17] H. Gerischer, "The impact of semiconductors on the concepts of electrochemistry," Electrochimica Acta, vol. 35, pp. 1677-1699, 1990.

[18] W. Schmickler, Grundlagen der Elektrochemie, Vieweg, Wiesbaden, Germany, 1996.

[19] M. S. Kilic, M. Z. Bazant, and A. Ajdari, "Steric effects in the dynamics of electrolytes at large applied voltages. I. Doublelayer charging," Physical Review E, vol. 75, no. 2, Article ID 021502, 16 pages, 2007.

[20] S. Roessler, R. Zimmermann, D. Scharnweber, C. Werner, and $\mathrm{H}$. Worch, "Characterization of oxide layers on Ti6Al4V and titanium by streaming potential and streaming current measurements," Colloids and Surfaces B, vol. 26, no. 4, pp. 387395, 2002.

[21] Y. Yang, R. Cavin, and J. L. Ong, "Protein adsorption on titanium surfaces and their effect on osteoblast attachment," Journal of Biomedical Materials Research A, vol. 67, no. 1, pp. 344-349, 2003.

[22] E. Eisenbarth, D. Velten, M. Müller, R. Thull, and J. Breme, "Biocompatibility of $\beta$-stabilizing elements of titanium alloys," Biomaterials, vol. 25, no. 26, pp. 5705-5713, 2004.

[23] P. Tengvall and I. Lundström, "Physico-chemical considerations of titanium as a biomaterial," Clinical Materials, vol. 9, pp. 115134, 1992. 
[24] M. K. Ridley, V. A. Hackley, and M. L. Machesky, "Characterization and surface-reactivity of nanocrystalline anatase in aqueous solutions," Langmuir, vol. 22, no. 26, pp. 10972-10982, 2006.

[25] T. Hanawa, "In vivo metallic biomaterials and surface modification," Materials Science and Engineering A, vol. 267, no. 2, pp. 260-266, 1999.

[26] F. P. Fehlner and N. F. Mott, "Low-temperature oxidation," Oxidation of Metals, vol. 2, no. 1, pp. 59-99, 1970.

[27] S. G. Steinemann, "Titanium-the material of choice?" Periodontol 2000, vol. 17, pp. 7-21, 1998.

[28] A. Stogryn, "Equations for calculating the dielectric constant of saline water (correspondence)," IEEE Transactions on Microwave Theory and Techniques, vol. 19, pp. 733-736, 1971.

[29] E. R. Nightingale, "Phenomenological theory of ion solvation. Effective radii of hydrated ions," Journal of Physical Chemistry, vol. 63, no. 9, pp. 1381-1387, 1959.

[30] S. Trasatti, "Work function, electronegativity, and electrochemical behaviour of metals. III. Electrolytic hydrogen evolution in acid solutions," Journal of Electroanalytical Chemistry, vol. 39, no. 1, pp. 163-184, 1972.

[31] M. A. Butler and D. S. Ginley, "Prediction of flatband potentials at semiconductor-electrolyte interfaces from atomic electronegativities," Journal of the Electrochemical Society, vol. 125, no. 2, pp. 228-232, 1978.

[32] R. Thull, "Physicochemical principles of tissue material interactions," Biomolecular Engineering, vol. 19, no. 2-6, pp. 43-50, 2002.

[33] S. Tanaka, "Performance simulation for dye-sensitized solar cells: toward high efficiency and solid state," Japanese Journal of Applied Physics, vol. 40, pp. 97-107, 2001.

[34] M. R. Oberholzer, N. J. Wagner, and A. M. Lenhoff, "Grand canonical Brownian dynamics simulation of colloidal adsorption," The Journal of Chemical Physics, vol. 107, no. 21, pp. 91579167, 1997. 

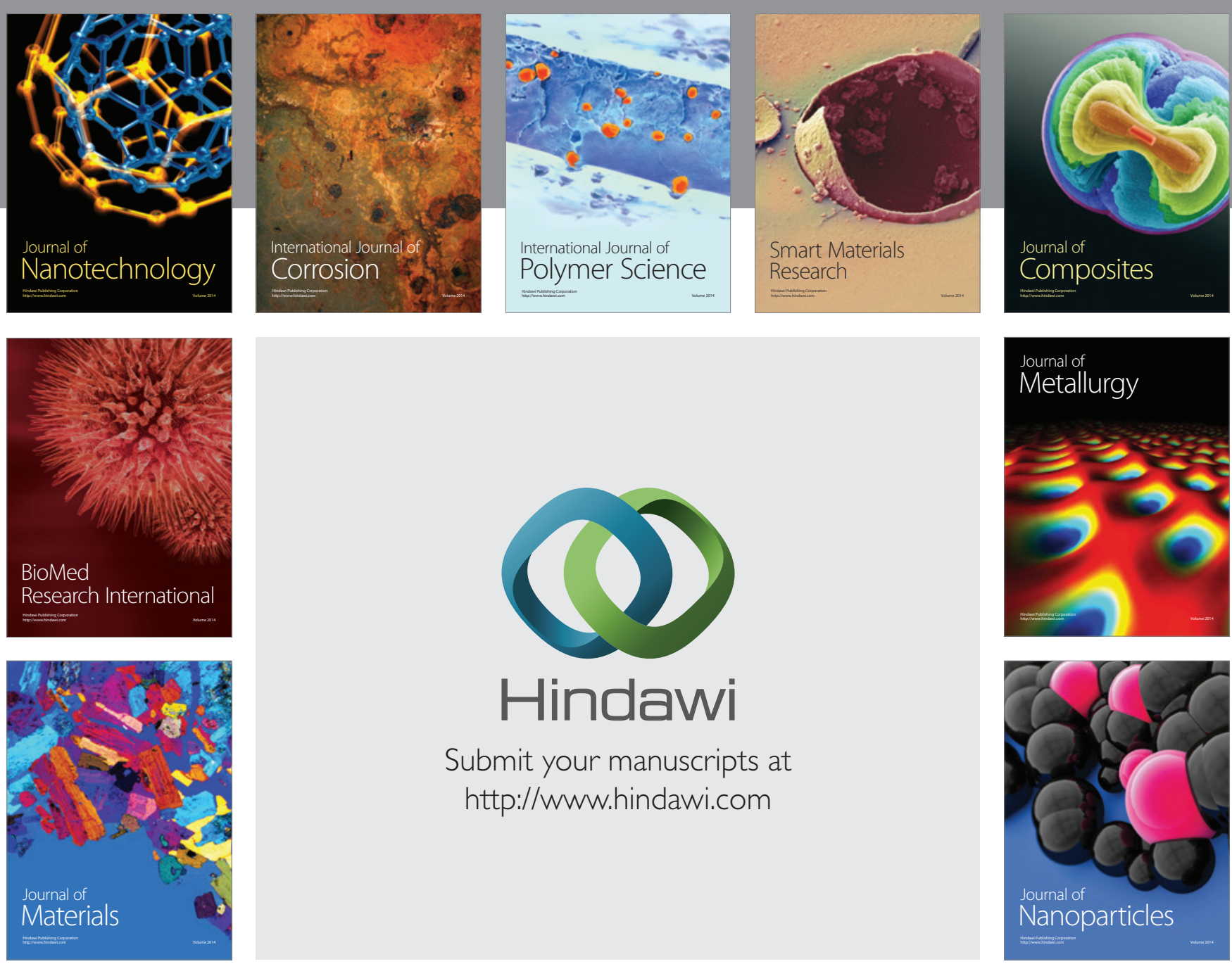

Submit your manuscripts at http://www.hindawi.com
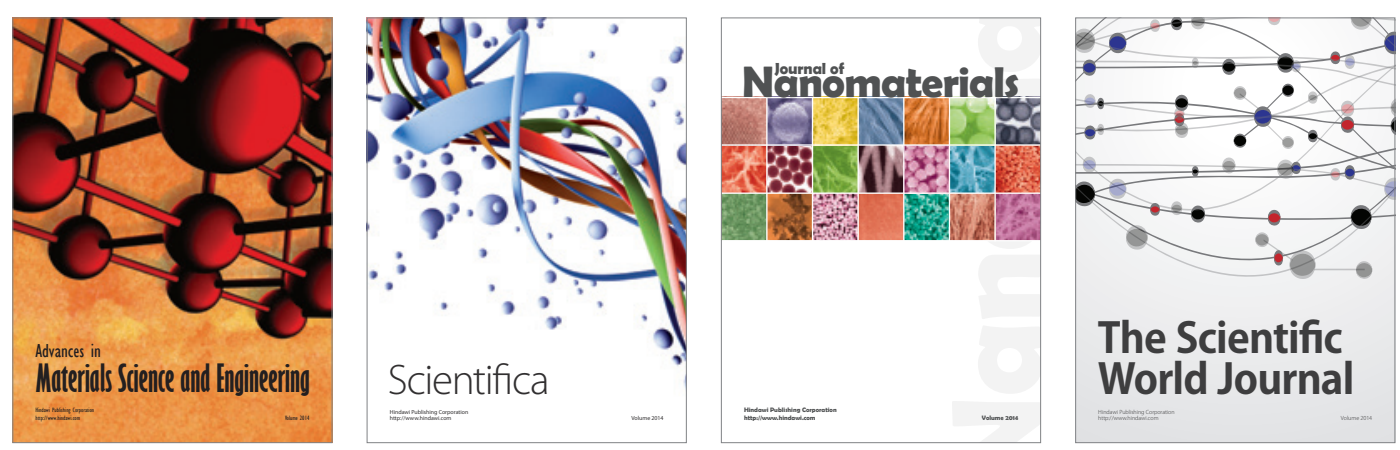

\section{The Scientific World Journal}
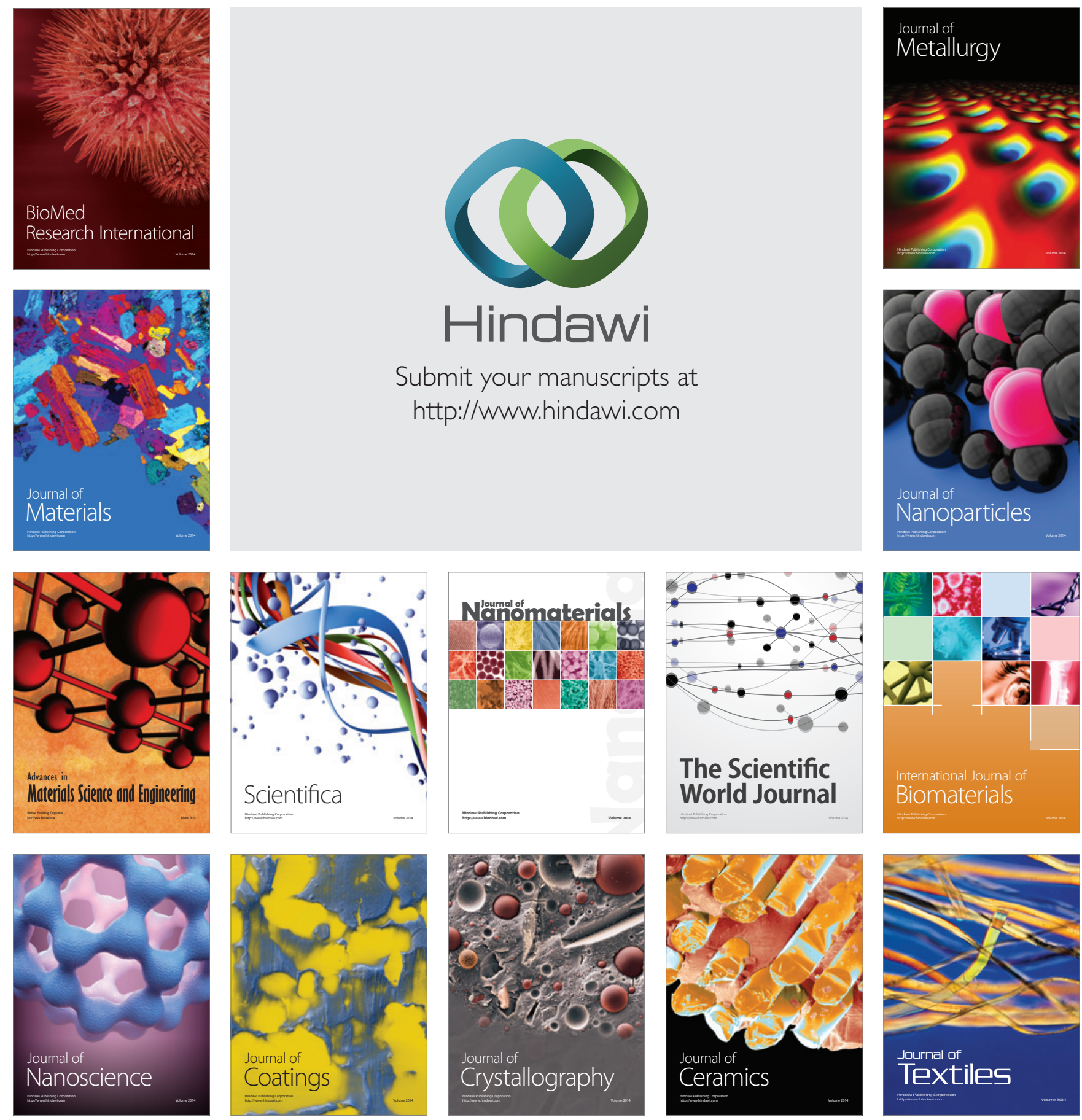